\title{
Activity of Entrectinib in a Patient With the First Reported NTRK Fusion in Neuroendocrine Cancer
}

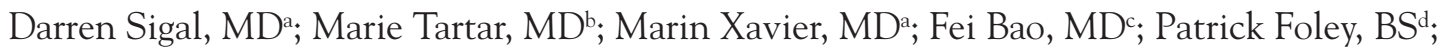 \\ David Luo, MPH'; Jason Christiansen, $\mathrm{PhD}^{\mathrm{d}}$; Zachary Hornby, MS, MBA ${ }^{\mathrm{d}}$; Edna Chow Maneval, $\mathrm{PhD}^{\mathrm{d}}$; \\ and Pratik Multani, MD, MS ${ }^{\mathrm{d}}$
}

\begin{abstract}
Despite advances in genomic analysis, the molecular origin of neuroendocrine tumors (NETs) is complex and poorly explained by described oncogenes. The neurotrophic TRK family, including NTRK1, 2, and 3, encode the proteins TRKA, TRKB, TRKC, respectively, involved in normal nerve development. Because NETs develop from the diffuse neuroendocrine system, we sought to determine whether NTRK alterations occur in NETs and whether TRK-targeted therapy would be effective. A patient with metastatic well-differentiated NET, likely of the small intestine, was enrolled on the STARTRK2 trial (ClinicalTrials.gov identifier: NCT02568267) and tissue samples were analyzed using an RNASeq next-generation sequencing platform. An ETV6:NTRK3 fusion was identified and therapy was initiated with the investigational agent entrectinib, a potent oral tyrosine kinase inhibitor of TRKA, TRKB, and TRKC. Upon treatment with entrectinib, the patient experienced rapid clinical improvement; his tumor response was characterized by initial tumor growth and necrosis. This is the first report of an NTRK fusion in NETs. Our patient's response to entrectinib suggests that NTRK fusions can be important in the pathogenesis of NETs. Recent DNA-based genomic analyses of NETs may have missed NTRK fusions due its large gene rearrangement size and multiple fusion partners. The tumor's initial pseudoprogression may represent a unique response pattern for TRK-targeted therapies. An effort to characterize the prevalence of NTRK fusions in NETs using optimal sequencing technology is important.
\end{abstract} doi: 10.6004/jnccn.2017.7029

\section{Background}

Neuroendocrine tumors (NETs) have been a mysterious group of neoplasms since their initial description by Dr. Oberndorfer in 1907, ${ }^{1}$ who reported on a tumor with the morphologic features of a carcinoma but indolent behavior, coining the term "karzinoide." With a median survival of only 33 months, patients with metastatic NETs have a poorer prognosis than previously realized. NETs have a prevalence of 35 per 100,000 individuals and have experienced a 5 -fold increase in incidence over the past 30 years, making effective NET therapy an important unmet medical need. ${ }^{2}$ Contemporary understanding of NETs has been clouded by divergent

From a Division of Hematology/Oncology, bepartment of Radiology, and 'Department of Pathology, Scripps Clinic, La Jolla; and 'Ignyta, Inc., San Diego, California.

Submitted May 11, 2017; accepted for publication September 1, 2017. Mr. Luo and Hornby and Drs. Christiansen, Chow Maneval, and Multani have disclosed that they are employed by and own equity in Ignyta. The remaining authors have disclosed that they have no financial interests, nomenclature, staging, and grading systems. NETs can form anywhere within the diffuse neuroendocrine system and have been characterized by their embryologic origin and their functionality-their ability to release bioactive molecules. NETs are also classified according to organ of origin, including small intestine (SI-NET) and pancreas (PNET). ${ }^{3}$ It is not surprising that the molecular pathogenesis of NETs remains elusive with this degree of clinical heterogeneity.

Many investigations into the tumorigenesis of NETs have focused on SI-NETs and PNETs, but their molecular pathology is still poorly understood. Definitive culpable oncogenic drivers are lacking even though these cancers

arrangements, affiliations, or commercial interests with the manufacturers of any products discussed in this article or their competitors.

The patient described in this case report signed an informed consent form and was enrolled in the STARTRK-2 (RXDX-101-02) trial sponsored by Ignyta, Inc. at Scripps Clinic (IRB Number: IRB-15-6698).

Correspondence: Darren Sigal, MD, Division of Hematology/Oncology, Scripps Clinic, 10666 North Torrey Pines Road, MS312, La Jolla, CA 92037. E-mail: sigal.darren@scrippshealth.org 
Sigal et al

have a stable genome with low rates of somatic mutations and copy number variations. ${ }^{4}$ Early chromosomal analyses frequently identified recurring chromosome 18 deletions in SI-NETs, and additional losses were seen in chromosomes 9, 13, 16, and 21. Additionally, amplifications were seen in chromosomes 4 , 5, 7, 14, and 20.4-7 PNETs contained a greater number of chromosomal aberrations that generally differed from SI-NETs, most importantly with alterations in chromosome 3 and loss of chromosome 11.,8-10 Candidate tumor suppressor genes are known to occur on deleted chromosomes, but second-hit events have been inconsistent. For example, the mechanism for CDKN2A (chromosome 9p) inactivation has been attributed to allelic deletion, promoter methylation, and somatic mutation. ${ }^{6}$ In contrast, the second copy of SMAD4 (chromosome 18) was not mutated and had normal protein expression in SI-NETs, suggesting additional tumor suppressors exist on chromosome $18 .^{7}$ Multiple other potential oncogenic pathways in SI-NETs have been described. ${ }^{11}$ Next-generation sequencing (NGS) of 48 SI-NETs was able to group nonsynonymous mutations and chromosomal alterations into 3 cancer-related pathways: $\mathrm{mTOR}, \mathrm{TGF}-\beta$, and the SRC oncogene. ${ }^{4}$

As opposed to SI-NETs, investigation into PNETs has revealed promising oncogenes. MEN1 was first identified as the gene responsible for MEN1 syndrome-related PNETs. ${ }^{12}$ Somatic mutations with deleted MEN1 were subsequently found to occur in $30 \%$ of sporadic PNETs. Because $80 \%$ of PNETs had abnormal cellular localization of menin, the gene product of MEN1, additional signaling and transit factors are also likely important. ${ }^{13}$ Wholeexome sequencing of 68 sporadic PNETs confirmed MEN1 mutations in 44\%, as well as newly identifying DAXX and ATRX mutations in $43 \%$ of specimens. ${ }^{14}$ Alternative full exon sequencing methodologies have confirmed the prevalence of mutations in MEN1, DAXX, and ATRX in 59\%, 33\%, and 26\% of PNETs, respectively, and less frequently in other potentially actionable mutations. ${ }^{15}$ Recently published whole-genome sequencing (WGS) of 102 PNETs described 4 pathways commonly altered in PNETs: DNA damage repair, chromatin remodeling, telomere maintenance, and, similar to SI-NETs, mTOR pathway activation. ${ }^{16}$
This report presents the first NTRK gene translocation identified in a patient with metastatic welldifferentiated NET, likely of the small intestine.

\section{Case Report}

A 28-year-old previously healthy man presented with progressive low back pain and an 11-kg weight loss over the previous year. He was a never-smoker, occasional alcohol drinker, and an avid runner. $\mathrm{He}$ had no relevant family history for cancer.

Laboratory workup revealed an elevated alkaline phosphatase level of $406 \mathrm{U} / \mathrm{L}$ (normal range, 38-126 $\mathrm{U} / \mathrm{L}$ ), and a right upper quadrant ultrasound noted echogenic masses in the liver. A CT scan identified low-density liver lesions, adrenal masses, bulky retroperitoneal and pelvic lymphadenopathy, and diffuse bony disease. Lumbar MRI showed diffusely abnormal marrow signal, with expansion of multiple vertebral bodies producing epidural encroachment. FDGPET imaging revealed widespread FDG uptake in the known areas of disease (Figure 1A). CT-guided biopsy of the left iliac bone revealed a well-differentiated
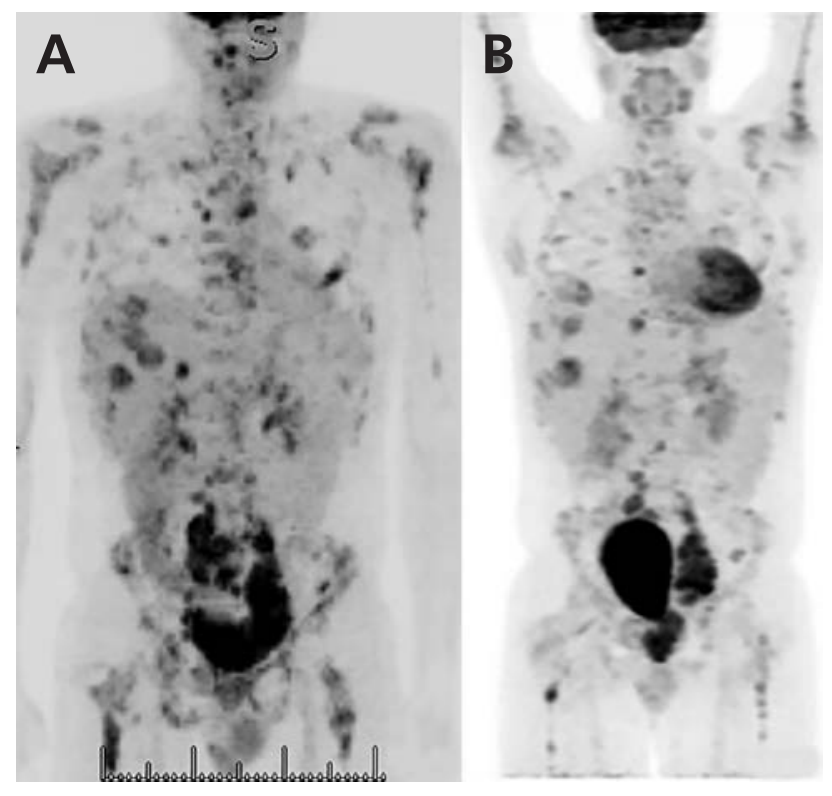

Figure 1. (A) Coronal PET maximum intensity projection (MIP) image shows the bulkiest and most intensely hypermetabolic disease to correspond with bulky iliac lymphadenopathy, with a large left pelvic sidewall confluent mass. Multiple hypermetabolic liver lesions are visible, and innumerable lesions are seen throughout the skeleton. (B) MIP PET scan after 2 cycles of entrectinib shows improvement compared with the presentation study, with previously confluent bony disease clearly improved (compare right proximal femur) and decreased bulk and intensity of activity of bulky pelvic lymphadenopathy. The most intense activity now seen in the pelvis is the elongated bladder, deviated to the right. 
NET, possibly from a lower gastrointestinal source. Findings of repeat CT-guided core biopsy of a right iliac soft tissue mass to confirm the absence of a highgrade component were consistent with those of the initial biopsy (Figure 2); there was no mitosis or necrosis identified in the tumor, and the $\mathrm{Ki}-67$ proliferative index was $1.2 \%$ by counting 500 nucleated tumor cells. Immunohistochemical (IHC) stains showed that the tumor cells were positive for synaptophysin, chromogranin, CDH17, CK20, CK19, and villin, and negative for TTF1, CDX-2, and CK7. These findings support the diagnosis of metastatic well-differentiated NET, grade 1, most likely of small intestine origin. An Indium-111 octreotide scan (Octreoscan) showed intensely increased scintigraphic activity localizing to bones, liver, and bulky retroperitoneum, and pelvic adenopathy (Figure 3A). Serum chromogranin A level was normal at $29 \mathrm{ng} / \mathrm{mL}$ (normal range, $<95 \mathrm{ng} /$ mL). 5-HIAA measured from a 24-hour urine collection was normal at $7 \mathrm{mg} / \mathrm{d}$ (normal range, $0-15 \mathrm{mg} / \mathrm{d}$ ). Serotonin was normal at $117 \mathrm{ng} / \mathrm{mL}$ (normal range, 50-200 ng/mL).

The patient received 3,000 cGy of palliative radiotherapy to L1-S3 in 10 fractions for pain control. He was then treated with combination temozolomide and capecitabine, but CT image showed progressive disease after 3 cycles. His tumor was analyzed with an RNA-based NGS platform. ${ }^{17}$ An interchromosomal gene rearrangement resulting in a fusion of exon 4 of ETV6 with exon 14 of NTRK3

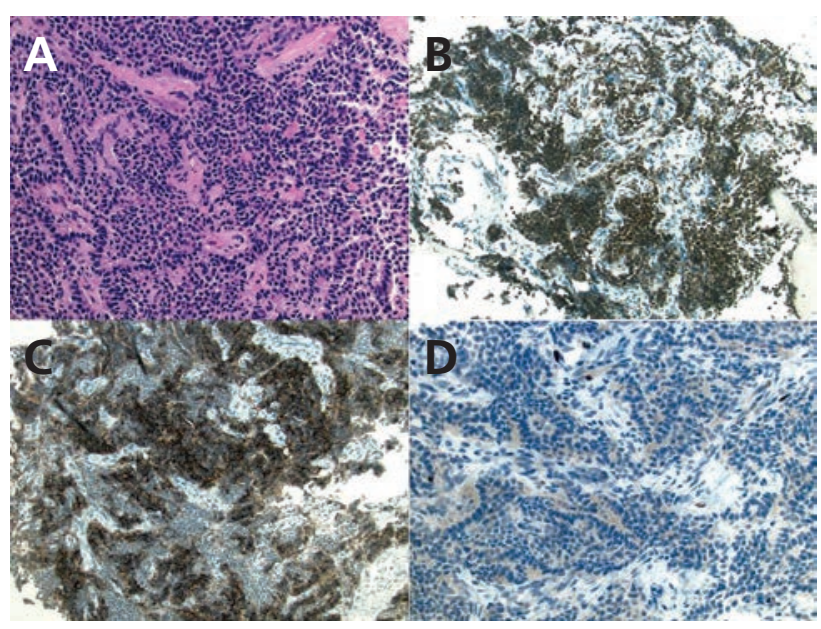

Figure 2. Metastatic well-differentiated neuroendocrine tumor, grade 1: (A) hematoxylin-eosin, original magnification $\times 200$; (B) synaptophysin immunostain, original magnification x100; (C) CDH17 immunostain, original magnification $\times 100$; and (D) Ki-67 labeling of $1.3 \%$ by immunohistochemistry (original magnification $\times 200$ ).

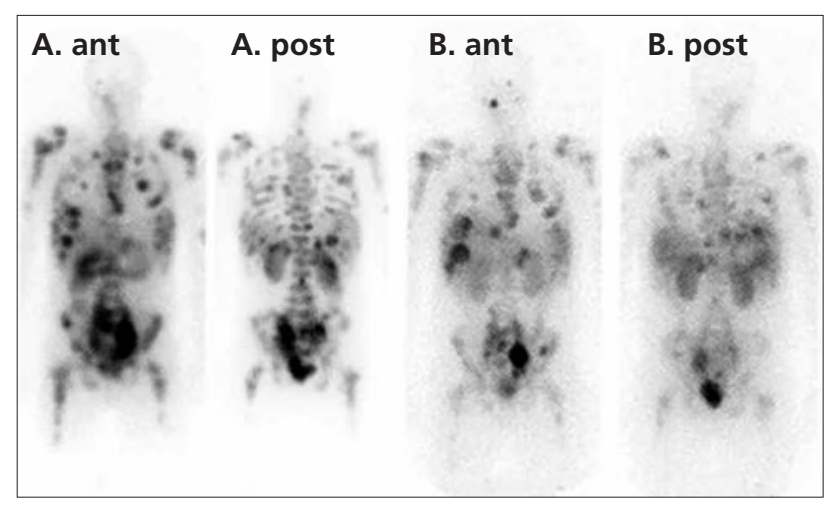

Figure 3. (A) Octreoscan imaging at presentation (anterior [ant] and posterior [post], planar) also shows the widespread distribution of disease, demonstrating the extensive skeletal metastases and bulky pelvic lymphadenopathy. (B) Octreoscan (ant and post, planar) after 6 cycles of entrectinib shows significant burden of disease, including in the liver, bones, and pelvis, but significant improvement compared with the staging Octreoscan. The largest and most active foci corresponds with the bulky left pelvic sidewall and presacral masses.

(ETV6:NTRK3) was identified. Based on the NTRK fusion, the patient was enrolled in the STARTRK2 trial (ClinicalTrials.gov identifier: NCT02568267), and therapy was initiated with the investigational agent entrectinib, a potent oral tyrosine kinase inhibitor of TRKA, TRKB, and TRKC.

After cycle 1 (28 days), the patient reported significant improvement in pain and energy levels, and also had a $6-\mathrm{kg}$ weight gain. Interestingly, multiple lesions in the liver and pelvis had grown, but RECIST measurements noted stable disease with a $14.1 \%$ increase in the sum of target lesion diameters. One liver lesion that increased from 4.6 to $6.4 \mathrm{~cm}$ in greatest diameter had a new cystic transformation. After cycle 2, FDG-PET/CT results showed progressive enlargement of the liver masses and of the pelvic and presacral adenopathy. RECIST measurements documented progressive disease with a $22.8 \%$ increase in the sum of target lesion diameters. However, the areas of tumor growth had lower density on CT and decreased FDG-uptake, indicating tumor necrosis. For example, the left pelvic sidewall mass had a decrease in maximum standardized uptake value $\left(\mathrm{SUV}_{\max }\right)$ from 9.9 to 6.1. There was also clear improvement in the skeleton (Figures $1 \mathrm{~B}$ and $4 \mathrm{~A}$, B). Due to the patient's significant clinical improvement and evidence of tumor necrosis explaining progressive disease per RECIST criteria, the sponsor and principal investigator agreed the patient should continue on study. Follow-up CT in subsequent months showed continued shrinkage of the pelvic nodal 


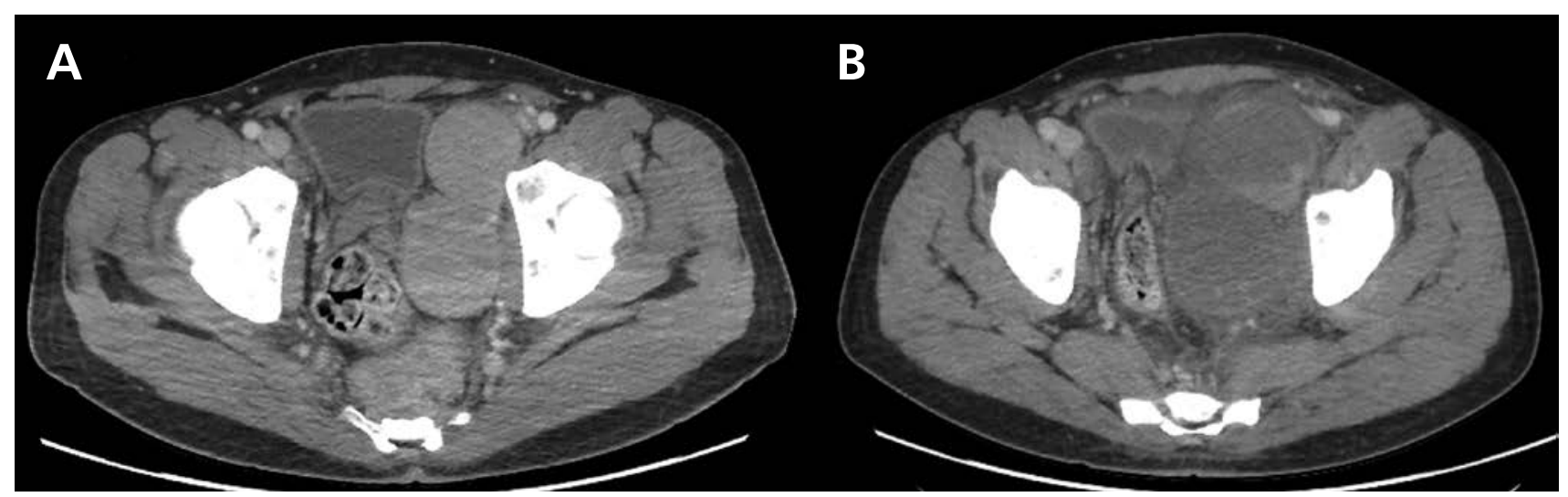

Figure 4. (A) Enhanced axial abdominal CT obtained after an initial trial of combination temozolomide and capecitabine chemotherapy shows bulky enhancing left pelvic sidewall and presacral nodal masses. (B) Enhanced axial abdominal CT at the same level, after 1 cycle of entrectinib, shows a dramatic change in appearance of left pelvic sidewall masses, which appear swollen and measure larger, but lower in density.

masses. An Octreoscan obtained 6 months after entrectinib initiation confirmed significant reduction in overall disease burden, with reduced but persistent activity in the pelvic masses (Figure 3B). Although the bulkiest disease sites in the pelvis and skeleton showed clear improvement, some liver metastases responded whereas others had not. The patient had a stabilized $12 \mathrm{-kg}$ weight gain, normalized energy level, and markedly reduced pain control requirements. He had not experienced any notable adverse events. After 12 months on entrectinib therapy, the patient had objective disease progression on CT imaging and was removed from the trial.

\section{Discussion}

The neurotrophic TRK family includes NTRK1, 2, 3 and encodes the tropomyosin receptor kinase receptors, TRKA, TRKB, and TRKC, respectively. These proteins are involved in normal nerve development, survival, and functionality. ${ }^{18}$ In the wild-type setting, a cognate ligand induces TRK homodimerization stimulating transphosphorylation of the kinase domain and activation of one of several pathways, including RAS-MAPK, mTOR, and PLC $\gamma$-PKC. NTRK translocations result in constitutively active protein fusions and are oncogenic in in vitro and in vivo models. ${ }^{18}$ Due to its genesis from the diffuse neuroendocrine system, we decided to screen patients with neuroendocrine cancers for an NTRK gene translocation. Of 15 patients screened thus far, we identified the first patient with a neuroendocrine cancer with an NTRK gene translocation,
ETV6:NTRK3. This is significant because the molecular etiology of NETs remains unclear and NTRK alterations may play a key role in NETs. Our patient's protracted response to entrectinib, a novel TRK-targeting therapy, supports this notion.

NTRK uses mTOR signaling that is also a common oncogenic pathway in both SI-NETs and PNETs. However, NTRK alterations have not been described in recent NGS studies of NETs. These reports have revealed known (notably MEN1, PTEN, TSC1, TSC2, PIK3CA, TP53, ARID1A, CDKN2A, CDKN2B) and previously unknown oncogenes (DAXX and ATRX), but NTRK translocations have been absent. ${ }^{4,14,15}$ Two main possibilities exist to explain these results. First, NTRK may be a rare oncogenic driver in NETs and go undetected in a small sample size. In addition, NETs from different organ systems generally contain unique tumorigenic alterations. Our patient's NET likely emanated from the small intestine, and NTRK alterations may possibly be important only in SI-NETs. This makes the sample size issue even more critical given that only one NGS study was conducted in SI-NETs and it included only 48 specimens. Second, NTRK translocations may not be reliably detected by targeted DNA sequencing NGS platforms. NTRK gene alterations have a promiscuous tendency for gene fusion partners, with at least 25 different gene translocations reported in at least 11 malignancies. ${ }^{19}$ Shorter read lengths, from both technology and as a result of formalin-fixed paraffin-embedded (FFPE) tissue preparation methods, and targeted enrichment may interfere with identification of large gene rearrangements 
or fusion partners outside of the surveyed genomic region.

It has been reported that WGS and RNA-seq NGS platforms may better identify gene translocations similar to those of NTRK. ${ }^{20}$ Our patient's tumor was analyzed using an RNA-based anchored multiplex PCR NGS assay. ${ }^{17,21}$ The test methodology does not require de novo knowledge of the fusion partner and is optimized to run with shorter fragments that are typically isolated from FFPE tissue specimens. Because the fusion is measured in RNA, challenges to identifying fusions that arise from tiling across large intronic spaces (the intron 13 preceding this specific result spans $\approx 93 \mathrm{~kb}$ ) or the potential for biological modifications after DNA processing are reduced. An WGS study of 102 patients with PNETs, that included RNA-seq in 30, did not identify a NTRK translocation, but did describe a novel mTOR pathway gene alteration in DEPDC5 in 2 patients $(1.9 \%) .{ }^{16}$ This suggests that a larger sample size may still reveal rare NTRK translocations in PNETs.

Several companies have TKIs targeting NTRK translocations in clinical development. Our patient was treated with entrectinib and quickly experienced a dramatic clinical benefit. Among 25 patients with a variety of malignancies all containing either NTRK, ROS1, or ALK gene fusions, who were naïve to prior inhibitors of the fusions, and who received an active dose of entrectinib, an overall response rate of $79 \%$ was observed, including significant tumor regression in all tumors with NTRK alterations, including ETV6:NTRK3 translocations. Responses were rapid, often occurring within 1 month of drug initiation, and were protracted. ${ }^{22}$ In contrast to typical response criteria, our patient's imaging studies demonstrated unique salutary changes, with early tumor size increase associated with tumor necrosis confirmed by photopenia on FDG-PET scanning. These imaging features are similar to the pseudoprogression noted in patients with imatinib-responsive gastrointestinal stromal tumors, explained by intratumoral hemorrhage, necrosis, or myxoid degeneration. $.^{23} \mathrm{Be}-$ cause only 25 entrectinib-treated patients evaluable for response have been reported, this novel pattern of response may emerge as an important outcome and should inform clinical trial design and response end points.

It should be noted that, despite the dramatic changes overall, some of the patient's liver lesions were unaffected, possibly reflecting the heterogeneous nature of NETs. This may be seen in the discordance between the lower $\mathrm{Ki}-67$ proliferation index of $<2 \%$ and positive Octreoscan associated with lower-grade NETs versus the diffuse FDG-PET positivity generally found in higher-grade NETs. It is unclear whether our patient's rapid and protracted response occurred because of a higher-grade component to his tumor or simply due to the presence of the NTRK translocation regardless of tumor grade.

\section{Conclusions}

As malignancies are increasingly characterized by even rarer genetic events, a dedicated effort to characterize the prevalence of NTRK alterations in NETs using optimal sequencing technology is very important. Although NETs from different organ systems have differential molecular signatures, certain oncogenic pathways are shared, such as mTOR, and NTRK alterations should be sought in all NET subtypes. Finally, NTRK-targeted therapies may produce unique response patterns that should be assessed in ongoing clinical trials.

\section{Acknowledgments}

The authors wish to thank Janet Shannon for her excellent study coordinator support for this trial.

\section{References}

1. Oberndorfer S. Carinoid tumors of the small intestine [in German]. Frankfurter Zeitschrift für Pathologie 1907;1:426-429.

2. Yao JC, Hassan M, Phan A, et al. One hundred years after "carcinoid": epidemiology of and prognostic factors for neuroendocrine tumors in 35,825 cases in the United States. J Clin Oncol 2008;26:3063-3072.

3. Klimstra DS, Beltran H, Lilenbaum R, Bergsland E. The spectrum of neuroendocrine tumors: histologic classification, unique features and areas of overlap. Am Soc Clin Oncol Educ Book 2015:92-103.

4. Banck MS, Kanwar R, Kulkarni AA, et al. The genomic landscape of small intestine neuroendocrine tumors. J Clin Invest 2013;123:2502-2508.

5. Kim DH, Nagano Y, Choi IS, et al. Allelic alterations in well-differentiated neuroendocrine tumors (carcinoid tumors) identified by genome-wide single nucleotide polymorphism analysis and comparison with pancreatic endocrine tumors. Genes Chromosomes Cancer 2008;47:84-92.

6. Kulke MH, Freed E, Chiang DY, et al. High-resolution analysis of genetic alterations in small bowel carcinoid tumors reveals areas of recurrent amplification and loss. Genes Chromosomes Cancer 2008;47:591-603.

7. Löllgen RM, Hessman O, Szabo E, et al. Chromosome 18 deletions are common events in classical midgut carcinoid tumors. Int J Cancer 2001;92:812-815.

8. Amato E, Barbi S, Malpeli G, et al. Chromosome 3p alterations in pancreatic endocrine neoplasia. Virchows Arch 2011;458:39-45. 
Sigal et al

9. Nagano Y, Kim DH, Zhang L, et al. Allelic alterations in pancreatic endocrine tumors identified by genome-wide single nucleotide polymorphism analysis. Endocr Relat Cancer 2007;14:483-492.

10. Oberg K. Genetics and molecular pathology of neuroendocrine gastrointestinal and pancreatic tumors (gastroenteropancreatic neuroendocrine tumors). Curr Opin Endocrinol Diabetes Obes 2009; 16:72-78.

11. Xavier S, Rosa B, Cotter J. Small bowel neuroendocrine tumors: from pathophysiology to clinical approach. World J Gastrointest Pathophysiol 2016;7:117-124.

12. Chandrasekharappa SC, Guru SC, Manickam P, et al. Positional cloning of the gene for multiple endocrine neoplasia-type 1. Science 1997;276:404407.

13. Corbo V, Dalai I, Scardoni M, et al. MEN1 in pancreatic endocrine tumors: analysis of gene and protein status in 169 sporadic neoplasms reveals alterations in the vast majority of cases. Endocr Relat Cancer 2010;17:771-783.

14. Jiao Y, Shi C, Edil BH, et al. DAXX/ATRX, MEN1, and mTOR pathway genes are frequently altered in pancreatic neuroendocrine tumors. Science 2011;331:1199-1203.

15. Raj NP, Soumerai T, Valentino E, et al. Next-generation sequencing (NGS) in advanced well differentiated pancreatic neuroendocrine tumors
(WD pNETs): a study using MSK-IMPACT [abstract]. J Clin Onco 2016;34(Suppl):Abstract e15661.

16. Scarpa A, Chang DK, Nones K, et al. Whole-genome landscape of pancreatic neuroendocrine tumours. Nature 2017;543:65-71.

17. Murphy DA, Ely HA, Shoemaker R, et al. Detecting gene rearrangements in patient populations through a 2-step diagnostic test comprised of rapid IHC enrichment followed by sensitive next-generation sequencing. Appl Immunohistochem Mol Morphol 2017;25:513-523.

18. Huang EJ, Reichardt LF. Trk receptors: roles in neuronal signal transduction. Annu Rev Biochem 2003;72:609-642.

19. Vaishnavi A, Le AT, Doebele RC. TRKing down an old oncogene in a new era of targeted therapy. Cancer Discov 2015;5:25-34.

20. Meldrum C, Doyle MA, Tothill RW. Next-generation sequencing for cancer diagnostics: a practical perspective. Clin Biochem Rev 2011;32:177-195.

21. Zheng Z, Liebers M, Zhelyazkova B, et al. Anchored multiplex PCR for targeted next-generation sequencing. Net Med 2014;20:1479-1484.

22. Drilon A, De Braud FG, Siena S, et al. Entrectinib, an oral pan-Trk, ROS1, and ALK inhibitor in TKI-naïve patients with advanced solid tumors harboring gene rearrangements: updated phase I results [abstract]. Cancer Res 2016;76(14 Suppl):Abstract CT007.

23. Choi H. Response evaluation of gastrointestinal stromal tumors. Oncologist 2008;13(Suppl 2):4-7. 